\title{
Diquarks and Exotic Spectroscopy
}

\author{
Robert Jaffe and Frank Wilczek \\ Center for Theoretical Physics, \\ Laboratory for Nuclear Science and Department of Physics, \\ Massachusetts Institute of Technology, \\ Cambridge, Massachusetts 02139 \\ MIT-CTP-3401
}

\begin{abstract}
We propose that the recently discovered $\Theta^{+}$baryon is a bound state of four quarks and an antiquark, containing two highly correlated $u d$-pairs. If so, the $\Theta^{+}$has positive parity, and it lies in an nearideally mixed $S U(3)_{f} \overline{\mathbf{1 0}}_{f} \oplus \mathbf{8}_{f}$. The Roper resonance and the $P_{11}(1710)$ fit naturally into this classification. We predict an isospin $3 / 2$ multiplet of $\Xi$ 's $(S=-2)$ with $J^{\Pi}=\frac{1}{2}^{+}$around 1750 $\mathrm{MeV}$. A search for manifestly exotic $\Xi^{+}$and $\Xi^{--}$in this mass range could provide a sharp test of our proposal. We predict that charm and bottom analogues of the $\Theta^{+}$are stable against strong decays.

PACS numbers: 12.38.-t, 12.39.-x, 14.20-c, 14.65.Bt
\end{abstract}

An exotic baryon, $\Theta^{+}(1540)$, with the quantum numbers of $K^{+} n$ has recently been reported by several groups [1, 2, 3]. The $\Theta^{+}$cannot be a three quark state. Its minimal quark content is $u u d d \bar{s}$, manifestly an exotic. It has hypercharge two and third component of isospin zero. The minimal SU(3)-flavor assignment is at the top of a $\overline{\mathbf{1 0}}_{f}$, also containing a $Y=1$ isodoublet $\left(N^{+}, N^{0}\right)$, a $Y=0$ isotriplet $\left(\Sigma^{+}, \Sigma^{0}, \Sigma^{-}\right)$, and a $Y=-1$ isospinquartet $\left(\Xi_{\frac{3}{2}}^{+}, \Xi_{\frac{3}{2}}^{0}, \Xi_{\frac{3}{2}}^{-}, \Xi_{\frac{3}{2}}^{--}\right)$. In this case the $\Theta^{+}$would be an isosinglet. The apparent absence of the $I_{3}=+1$, $\Theta^{++}$in $K^{+} p$ argues against $I=1$. We assume the $\Theta^{+}$ to be an isosinglet.

Exotics with the quark content of the $\Theta^{+}$have been proposed in the context of quark and bag models since the early days of QCD [4, 5]. Their possible importance

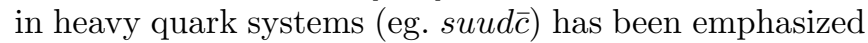
by Lipkin [6].

More recently the $\mathrm{SU}(3)$-flavor $\overline{\mathbf{1 0}}_{f}$ representation has emerged as an interesting feature of chiral soliton (Skyrme) models of baryons. Soon after the study of $\mathrm{SU}(2)$ chiral solitons by Adkins, Nappi, and Witten [7], three flavor generalizations led to predictions of a low lying $J=\frac{1}{2} \overline{\mathbf{1 0}}_{f}[8]$. As early as 1987 Praszalowicz predicted that the $Y=2$ isosinglet member of the $J=\frac{1}{2} \overline{\mathbf{1 0}}_{f}$ would lie near $1540 \mathrm{MeV}$ [9]. In 1997 Diakonov, Petrov, and Polyakov (DPP) not only predicted a $\Theta$ at about $1540 \mathrm{MeV}$, but also estimated its width at less than 15 $\mathrm{MeV}[10,11]$.

The discovery of a manifestly exotic baryon provides an opportunity to refine our understanding of quark dynamics at low energy, where it is not perturbative. In this Letter we propose that the $\Theta^{+}$can be usefully considered as a bound state of an antiquark with two highly correlated spin-zero $u d$-diquarks. This interpretation has some desirable features and makes predictions that distinguish it clearly both from the traditional uncorrelated quark model and from the chiral solition model.

Let us first discuss some general features of the dynamics of a $K^{+} n$ resonance. The $\Theta^{+}$appears to be remarkably narrow. It lies about $100 \mathrm{MeV}$ above $K^{+} n$ threshold at a center of mass momentum of $k=270$ $\mathrm{MeV}$. Taking a typical range of hadronic interactions $R$ of 1 fermi, the characteristic parameter $k R$ is about 1.4. Assuming isospin zero, the possible quantum numbers of the $\Theta^{+}$(in $K N$ scattering notation, $L_{I(2 J)}$ ) are $S_{01}, P_{01}$, $P_{03}, D_{03}, D_{05}, \ldots$ With $k R \approx 1.4$ only the $S$ or $P$ wave is likely, and there is some indication that the spin of the $\Theta^{+}$is $\frac{1}{2}[12$. At center of mass momentum $270 \mathrm{MeV}$ the kaon and neutron are not very relativistic. There are no other two-body hadronic channels coupling to $K^{+} n$ below $K \Delta$ threshold at $1725 \mathrm{MeV}$. Since there are no quark-antiquark annihilation graphs in $K^{+} n$ scattering (that is what it means to be exotic) one expects, and observes, that low energy $\mathrm{K}^{+} n$ scattering is dominated by ordinary exchange forces. This contrasts with non-exotic channels, for example $\pi N$, which are dominated by confined states that couple by $\bar{q} q$ annihilation, for example the $\Delta$. QCD allows $K^{+} n$ to couple to $q^{4} \bar{q}$ configurations with wavefunctions that differ markedly in space, color, and spin from the $K^{+} n$ scattering state. Without explicit introduction of such effects, one has no choice but to describe $K^{+} n$ scattering in the region of the $\Theta^{+}$in terms of (non-relativistic) potential scattering. Let us see where that leads. Attractive forces in the $s$-wave do not generate resonances. If they are attractive enough they produce bound states like the deuteron (or virtual states like the dineutron), if not, they modulate the phase of the scattering wavefunction in a smooth fashion. In higher partial waves attractive forces typically generate resonances through interplay between the attraction and the repulsive angular momentum barrier. The mass and width of the resonance are related through the range and 
depth of the potential. For a simple attractive potential (we used a square well) of range 1 fermi, the width of a $P$-wave resonance $100 \mathrm{MeV}$ above threshold is above $175 \mathrm{MeV}$. To produce a width of order $10 \mathrm{MeV}$ requires a range of about 0.05 fermi, which brings in a high energy scale. Thus the observed width of the $\Theta^{+}$suggests that its coupling to the $K^{+} n$ channel is suppressed by some additional dynamics beyond non-relativistic potential scattering.

Now let us discuss the predictions and reliability of the chiral soliton and the traditional (uncorrelated) quark models. In the chiral soliton model the $\left(\overline{\mathbf{1 0}}_{f}, \frac{1}{2}{ }^{+}\right)$is the third baryon $\mathrm{SU}(3)$-flavor multiplet in the same "rotational band" as the $\left(\mathbf{8}_{f}, \frac{1}{2}^{+}\right)$and $\left(\mathbf{1 0}_{f}, \frac{3}{2}^{+}\right)$. Like them it has positive parity. The successful prediction of a narrow $\frac{1}{2}^{+} \Theta$ near $1540 \mathrm{MeV}$ is remarkable, but there are reasons for reserve. At best this model approximates QCD in the $N_{c} \rightarrow \infty$ limit; in addition, it involves a poorly controlled truncation of the chiral lagrangian, adiabatic quantization of a system without separation of scales and - what is especially pertinent - in the three-flavor case, heavy reliance on chiral $S U(3) \times S U(3)$ symmetry, which is badly broken in Nature. It abounds with states that are exotic for $N_{c}=3$ and, since they are not observed in Nature, must be discarded as artifacts. Even the two flavor model predicts exotic baryons with $(I, J)=\left(\frac{5}{2}, \frac{5}{2}\right),\left(\frac{7}{2}, \frac{7}{2}\right) \ldots$. Difficulties that arise in $S U(3)$ chiral soliton models are discussed in Refs. 13.

The concepts of the quark model are more obviously grounded in microscopic QCD, and of course this model has had much success in describing the meson and baryon spectrum. The light quark mesons and baryons by and large fall neatly into the $\mathrm{SU}(3)$-flavor multiplets predicted by the quark model. In particular, the ground state multiplets of the $\bar{q} q$ and $q^{3}$ have the quantum numbers, including parity, expected of a collection of quarks (and antiquarks) in the same eigenstate of some mean field. Thus the lightest mesons - the pseudoscalar and vector nonets - have negative parity; and the lightest baryons (the $\left(\boldsymbol{8}_{f}, \frac{1}{2}\right)$ and the $\left.\left(\mathbf{1 0}_{f}, \frac{3}{2}\right)\right)$ have positive parity.

The nonet of light scalar $\left(J^{P C}=0^{++}\right)$mesons with masses below $1000 \mathrm{MeV}\left(f_{0}(600), f_{0}(980), a_{0}(980)\right.$, $\kappa(800$ ?)), however, does not seem to fit a $\bar{q} q$ description 14]. Instead, a heavier scalar nonet above $1000 \mathrm{MeV}$ seems to be largely $\bar{q} q$. The light scalars seem at least to have a significant component of $\bar{q} \bar{q} q[14$ dominated by a configuration where the pairs of quarks (and antiquarks) are separately correlated in a flavor, color, and spin antisymmetric state 15]. This diquark is a boson with color $\overline{\mathbf{3}}_{c}$, flavor $\overline{\mathbf{3}}_{f}$, and spin zero. A diquark and antidiquark neatly couple together to give a nonet of color singlet scalar mesons. The apparent fact that the $\bar{q} \bar{q} q q$ scalars are lighter than the $\bar{q} q$ scalars testifies to the strength of the diquark correlation. There is also evidence for strong diquark correlations in the baryon spectrum [16].
Attraction between quarks in the color $\overline{\mathbf{3}}_{c}$ channel has profound roots in microscopic QCD. Indeed, by bringing quarks together in this channel one halves the magnitude of their effective charge, and thus largely cancels the associated field energy. This idea is the basis color superconductivity in dense quark matter, a context in which it can be made quite rigorous 17.

With these considerations in mind let us now consider the $q^{4} \bar{q}$ system. In an uncorrelated quark model, in which all the quarks are in the ground state of a mean field, the ground state of $q^{4} \bar{q}$ has negative parity. This is a striking difference from the chiral soliton model, and from the correlated quark model we are advocating. The full $S U(3)_{f} \times S U(2)_{\text {spin }}$ content of this multiplet and bag model estimates of masses and decay couplings can be found in Ref. [5]. There are very many states in flavor $\mathbf{1}_{f}$, $\mathbf{8}_{f}, \overline{\mathbf{1 0}}_{f}, \mathbf{2 7}_{f}$, etc.. The lightest have the quark content $u u d d \bar{d}$ and $u u d d \bar{u}$ and would be expected to lie below the $\Theta^{+}$. There is no evidence for a $\frac{1}{2}^{-}$nucleon in this well explored region of the non-strange baryon spectrum. Furthermore, all the light negative parity baryons are well described as orbital excitations of $q^{3}$.

We propose instead the following picture of the $\Theta^{+}$and other $q^{4} \bar{q}$ baryons. The four quarks are bound into two spin zero, color and flavor $\overline{\mathbf{3}}$ diquarks. For our purposes we can regard the diquarks as composite bosons, $[u d]$, $[d s]$, and $[s u]$. The diquarks obey Bose statistics, but may be expected to experience a repulsive "Pauli blocking" interaction at short distances. In order to combine with the antiquark into a singlet, the two diquarks must combine into a color $\mathbf{3}$. Since the $\mathbf{3}$ is the antisymmetric part of $\overline{\mathbf{3}} \times \overline{\mathbf{3}}$, the diquark-diquark wavefunction must be antisymmetric with respect to the rest of its labels. For identical diquarks, like $[u d]^{2}$ only space labels remain, and we conclude that the lightest state must have a wavefunction antisymmetric under space exchange, i.e. with negative space-parity. When combined with the antiquark, the resulting $q^{4} \bar{q}$ state therefore has positive parity. The three unlike diquark pairs, $[u d][d s],[d s][s u]$, and $[s u][u d]$, can be antisymmetrized in flavor, and therefore can have symmetric, i.e. positive parity, spatial wavefunctions. However it is not unreasonable to expect that the blocking repulsion raises the energy of the spatially symmetric states. Evidence, albeit indirect, for Pauli blocking in the $s$-wave may also come from the apparent absence of a tightly bound $H$-dibaryon ([ud] [ds][su]). We hypothesize that this effect is strong enough to elevate the resulting negative parity nonet of $q^{4} \bar{q}$ states to masses high enough that their effects on the mesonbaryon $S$-wave, where they lie, are weak. In any case all these negative parity states have conventional quantum numbers $\left(\mathbf{8}_{f}\right.$ and $\left.\mathbf{1}_{f}\right)$ and contain at least one strange quark. Thus the $Y=1, I=\frac{1}{2}$ "nucleon" has the quark content $u u d s \bar{s}$ and would be heavy and weakly coupled to $\pi N$. We put these states aside.

The flavor symmetric, and therefore spatially antisym- 


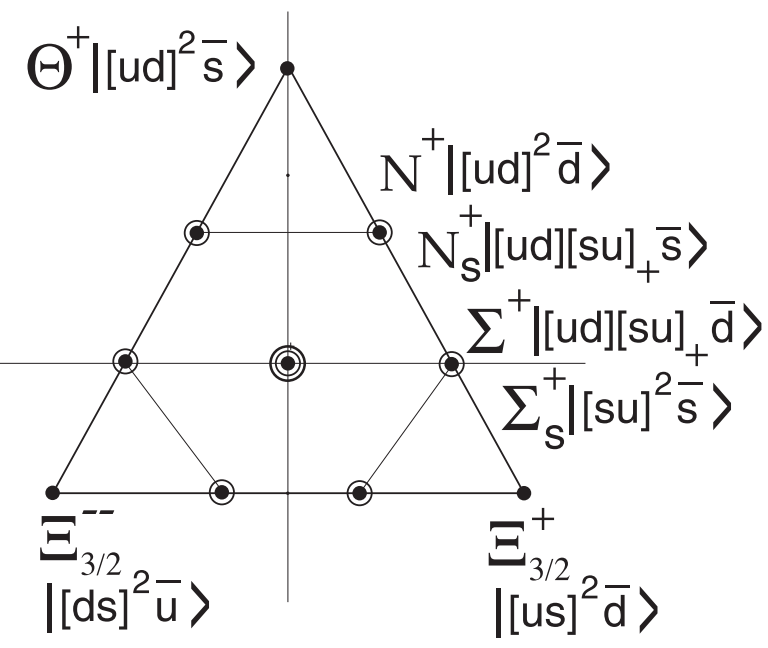

FIG. 1: Quark content of representative members of the $\left(q^{4} \bar{q}\right) \overline{\mathbf{1 0}}_{f}+\mathbf{8}_{f}$.

metric, two diquark states form an $S U(3)_{f} \overline{\mathbf{6}}_{f}$ : $[u d]^{2}$, $[u d][u s]_{+},[u s]^{2},[u s][d s]_{+},[d s]^{2}$, and $[d s][u d]_{+}$. When combined with the antiquark the result is a degenerate $S U(3)_{f} \mathbf{8}_{f} \oplus \overline{\mathbf{1 0}}_{f}$. The strange quark mass breaks $S(3)_{f}$ symmetry. In addition to the mass itself, the $[u d]$ diquark is more tightly bound than the $[u s]$ or $[d s]$ diquarks, an effect well-known from baryon spectroscopy [18. This effect can be related to the $\Sigma-\Lambda$ mass difference. It leads to a contribution $\alpha \equiv \frac{3}{4}\left(M_{\Lambda}-M_{\Sigma}\right) \approx 60 \mathrm{MeV}$ for every strange quark. A schematic Hamiltonian including $S U(3)$ violation is $H_{s}=M_{0}+\left(n_{s}+n_{\bar{s}}\right) m_{s}+n_{s} \alpha$. It will ideally mix the $\overline{\mathbf{1 0}}_{f}$ and $\boldsymbol{8}_{f}$ according to their strange quark content. Of course a more sophisticated treatment would allow for non-ideal mixing and perturb our mass estimates. The quark content of representative states in the $\overline{\mathbf{1 0}}_{f} \oplus \mathbf{8}_{f}$ is given in Fig. (11). The mass hierarchy of the states is summarized in Fig. (2) and compared with the chiral soliton model. The spatially antisymmetric wavefunction of the pairs of diquarks would likely correspond to angular momenum one. Thus the total angular momentum of the correlated $\left(q^{4} \bar{q}\right)$ system would be either $\frac{1}{2}$ or $\frac{3}{2}$. We assume that the $\frac{3}{2}^{+} \boldsymbol{8}_{f} \oplus \overline{\mathbf{1 0}}_{f}$ is elevated to somewhat higher energies, where the states fall apart quickly into mesons and baryons and do not produce prominent resonances. Of course, if our picture proves correct, a dynamical explanation for this $L S$ splitting must be found.

The $\mathbf{8}_{f}+\overline{\mathbf{1 0}}_{f}$ has many interesting features:

$\boldsymbol{\Theta}^{+}\left([\mathbf{u d}]^{2} \overline{\mathbf{s}}\right)$ : We identify this state with the $\Theta^{+}$. This fixes $M_{0}+m_{s} \approx 1540 \mathrm{MeV}$. It may be possible to explain the narrowness of the $\Theta^{+}$by the relatively weak coupling of the $K^{+} n$ continuum to the $[u d]^{2} \bar{s}$ state from which it differs in color, spin and spatial wavefunctions.

$\mathbf{N}\left([\mathbf{u d}]^{2} \overline{\mathbf{d}}\right)$ : This is the lightest particle in the $\mathbf{8}_{f}+\overline{\mathbf{1 0}}_{f}$, with mass $M_{0}$. It has the quantum number of the nu- cleon. It is tempting to identify this state with the otherwise perplexing Roper resonance, the $N(1440) P_{11}$. The assignment would explain the existence of the Roper and fixes $M_{0} \approx 1440 \mathrm{MeV}$. The $N(1440)$ is much broader than the $\Theta^{+}$. The greater phase space for $N(1440) \rightarrow N \pi$ compared to $\Theta^{+} \rightarrow K^{+} n,\left[p\left(\Theta^{+}\right) / p(N(1440)]^{3} \approx 3\right.$ helps, but does not nearly account for the width. Of course, in our picture the internal structure and grouptheoretic properties of $\Theta^{+}$and $N(1440)$ are quite different.

$\boldsymbol{\Sigma}\left([\mathbf{u d}][\mathbf{s u}]_{+} \overline{\mathbf{d}}\right)$ and $\boldsymbol{\Lambda}([\mathbf{u d}][\mathbf{d s}] \overline{\mathbf{d}}-[\mathbf{s u}][\mathbf{u d}] \overline{\mathbf{u}})$ : These $\frac{1}{2}^{+}$hyperon resonances should have masses, $M(\Sigma, \Lambda) \approx$ $M_{0}+m_{s}+\alpha \approx 1600 \mathrm{MeV}$, well above threshold for decay to $\Lambda(1116) \pi$ and $\Sigma(1192) \pi$. We expect them to be broader than the $\Theta^{+}$. The PDG identify $\Sigma$ "bumps" at $1480 \mathrm{MeV}$ and $1560 \mathrm{MeV}$. There is a well established $\Lambda(1600) P_{01}$ and $\Sigma(1660) P_{11}$ both with $J^{\Pi}=\frac{1}{2}^{+}$.

$\mathbf{N}_{\mathbf{s}}\left([\mathbf{u d}][\mathbf{s u}]_{+} \overline{\mathbf{s}}\right)$ : Although it has the same quantum numbers as the nucleon, this state has hidden strangeness (like the $\phi(1020)$ ) and is therefore heavier and should couple anomalously strongly to strange particles. We estimate $M\left(N_{s}\right) \approx M_{0}+2 m_{s}+\alpha \approx 1700 \mathrm{MeV}$. There is a candidate, the $N(1710) P_{11}$. If this assignment is correct, then the $N(1710)$ should couple more strongly to $N \eta$, $K \Lambda$, and $K \Sigma$ than currently suspected.

$\boldsymbol{\Sigma}_{\mathbf{s}}\left([\mathbf{s u}]^{2} \overline{\mathbf{s}}\right):$ Like the $N_{s}$, this state has hidden strangeness. It is predicted to be heavy, $M\left(\Sigma_{s}\right) \approx$ $M_{0}+3 m_{s}+2 \alpha \approx 1850 \mathrm{MeV}$, and to couple predominantly to $\Sigma \eta$ and $\Xi \bar{K}$. Since it couples weakly to $\bar{K} N$, it would have been difficult to see.

$\mathbf{\Xi}\left(\sqrt{\mathbf{2}}[\mathbf{u s}]^{2} \overline{\mathbf{u}}-[\mathbf{s u}][\mathbf{d s}] \overline{\mathbf{d}}\right)$ : This $\frac{1}{2}^{+}$cascade resonance should have mass $M(\Xi) \approx M_{0}+2 m_{s}+2 \alpha \approx 1750 \mathrm{MeV}$. Some $\Xi$ 's are known in this region, but none have established spin-parity assignments.

$\boldsymbol{\Xi}_{\frac{3}{2}}\left([\mathbf{u s}]^{\mathbf{2}} \overline{\mathbf{d}}\right)$ : This isospin $3 / 2$ multiplet contains two $\Xi$ 's with ordinary charge assignments, $\Xi_{\frac{3}{2}}^{0}$ and $\Xi_{\frac{3}{2}}^{-}$. In addition it includes the exotic $\Xi_{\frac{3}{2}}^{+}$and $\Xi_{\frac{3}{2}}^{--}$. These exotics should have mass near $1750 \mathrm{MeV}$. There is a clean $S U(3)$ relation between the matrix elements for $\Theta^{+} \rightarrow p K^{0} / n K^{+}$and $\Xi^{--} \rightarrow \Sigma^{-} K^{-} / \Xi^{-} \pi^{-}$. Adjusting for differences in phase space, the width of the $\Xi^{--}$ at a mass of $1750 \mathrm{MeV}$ should be about $50 \%$ greater than the width of the $\Theta^{+}$.

The spectrum of the $\mathbf{8}_{f} \oplus \overline{\mathbf{1 0}}_{f}$ correlated quark picture differs in several dramatic ways from the spectrum predicted by the chiral soliton model. The differences are highlighted in Fig. (2). The chiral soliton model predicts only a $\overline{\mathbf{1 0}}_{f}$. First order perturbation theory in $m_{s}$ predicts equal spacing between isomultiplets of decreasing hypercharge. None of the phenomena related to ideal mixing of the $\mathbf{8}_{f} \oplus \overline{\mathbf{1 0}}_{f}$ occur. In particular in the chiral soliton model: a) There is no $N$ lighter than the $\Theta^{+}$, therefore no candidate for the Roper resonance; b) The $N$ that is heavier than the $\Theta$ couples both to strange and non-strange channels. The $N_{s}$ in the correlated 


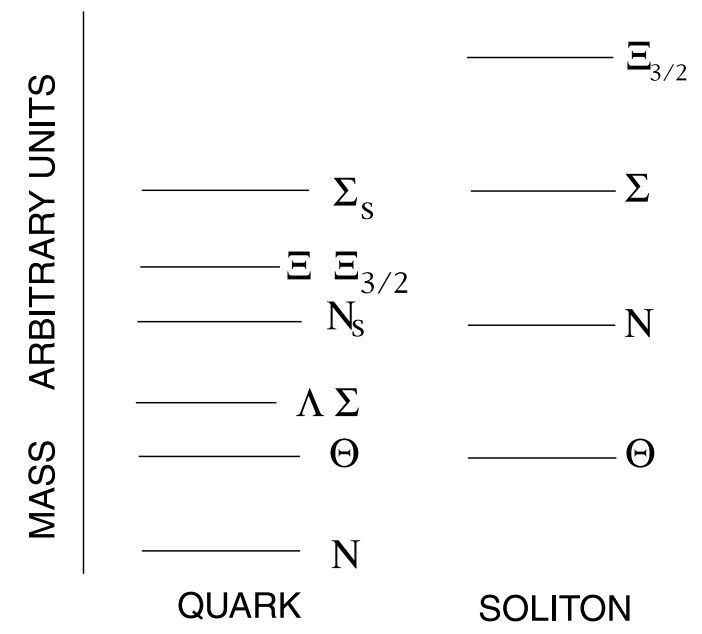

FIG. 2: Relative masses of states in the ideally mixed $\left(q^{4} \bar{q}\right) \overline{\mathbf{1 0}}_{f} \oplus \mathbf{8}_{f}$, compared with the mass hierarchy in the chiral soliton model.

quark picture should couple predominantly with hidden strangeness (like the $\phi(1020)$ or $f_{0}(980)$ ); c) The $\Xi$ states are heaviest - above $2 \mathrm{GeV}$. In the correlated quark picture they are close to the $N_{s}$ because they both contain two $s$-quarks. The prediction of light charge-exotic $\Xi$ 's is the most distinctive signature of our model; d) There is no second multiplet of $\Sigma$ 's with hidden strangeness and no $\Lambda$. The striking departure of predicted mass splittings in the chiral soliton model from an approximate additive quark mass formula arises from that model's powerful embodiment of chiral flavor $S U(3) \times S U(3)$.

Exotic channels ought to be accessible to numerical investigation using lattice gauge theory. The predicted resonances are above threshold, but not by much, so they should be visible in long-range correlators. By comparing different spatial and flavor trial wave functions, one could isolate the effect of the suggested (diquark) configurations. One could also bring the states closer to threshold, or perhaps even below it, by increasing quark masses, as in an analogous study of the scalar mesons [19].

Our picture suggests thatthe charm and bottom analogs of the $\Theta^{+}$may be stable against strong decays. The $\Theta_{c}^{0}=[u d]^{2} \bar{c}$ and $\Theta_{b}^{+}=[u d]^{2} \bar{b}$ differ from the $\Theta^{+}$ by the replacement $\bar{s} \rightarrow \bar{c}$ or $\bar{b}$. The cost of this can be estimated by comparing the masses of the $\Lambda(1116)$, $\Lambda_{c}(2285)$, and $\Lambda_{b}(5624 \pm 9)$. The $[u d]$ diquark in the $\Lambda$ is coupled to color $\overline{\mathbf{3}}$ and spin zero and so provides an environment for the $s$ quark nearly identical to the environment of the $\bar{s}$ quark in the $\Theta^{+}$(plausible in the limit of tight diquark binding). Therefore we estimate $M\left(\Theta_{c}^{0}\right)-M\left(\Theta^{+}\right)=M\left(\Lambda_{c}\right)-M(\Lambda)=1170 \mathrm{MeV}$. This yields $M\left(\Theta_{c}^{0}\right)=2710 \mathrm{MeV}$, which is $100 \mathrm{MeV}$ below threshold for the strong decay $\Theta_{c}^{0} \rightarrow p D^{-}$. Likewise, we estimate $M\left(\Theta_{b}^{+}\right)-M\left(\Theta^{+}\right)=M\left(\Lambda_{b}\right)-M(\Lambda)=4510 \pm 10$
$\mathrm{MeV}$, and therefore $M\left(\Theta_{b}^{+}\right)=6050 \pm 10 \mathrm{MeV}$, which is $165 \pm 10 \mathrm{MeV}$ below threshold for the strong decays $\Theta_{b}^{+} \rightarrow p B^{0}$ and $n B^{+}$. The sequence of binding energies, $-100 \mathrm{Mev}, 100 \mathrm{MeV}$, and $165 \mathrm{MeV}$, for the $\Theta^{+}, \Theta_{c}^{0}$, and $\Theta_{b}^{+}$reflects the non-linear dependence of the pseudoscalar meson $(K, D$, and $B)$ masses on the quark masses. The $\Theta_{c}^{0}$ could be produced, for example, in $\pi^{-} p \rightarrow \Theta_{c}^{0} D^{0}$ or $\Theta_{c}^{0} D^{+} \pi^{-}$or even in non-leptonic $B$-decays, such as $B^{0} \rightarrow \Theta_{c}^{0} \bar{p} \pi^{+}[20]$.

We have presented a description of $q^{4} \bar{q}$ baryons based on the strong color-spin correlation force that drives color superconductivity and may be responsible for the peculiarities of the light scalar mesons. A light positive parity $K^{+} n$ exotic is natural in this picture. So is an even lighter $\frac{1}{2}^{+}$nucleon, which we tentatively identify with the Roper resonance. Our description differs in important ways from the chiral soliton description of the $\Theta^{+}$, and our prediction of relatively light charge-exotic cascades should be testable in both numerical and accelerator experiments.

After submission of this Letter, evidence for an exotic $\Xi^{--}$was reported by the NA49 Collaboration 21], at a mass about $100 \mathrm{MeV}$ above our rough estimate.

\section{Acknowledgments}

We thank K. Hicks for correspondence and N. Samios for conversations. One of us (RLJ) would like to thank M. Praszalowicz for conversations. This work is supported in part by the U.S. Department of Energy (D.O.E.) under cooperative research agreements \#DFFC02-94ER40818.

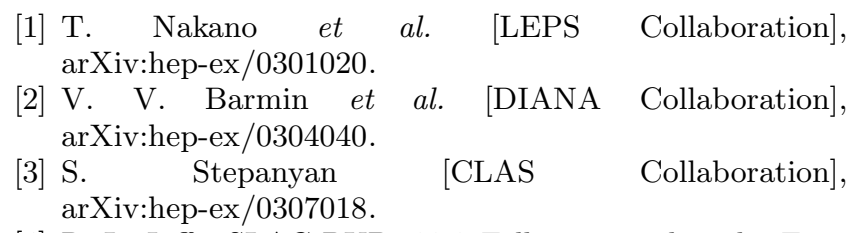

[4] R. L. Jaffe, SLAC-PUB-1774 Talk presented at the Topical Conf. on Baryon Resonances, Oxford, Eng., Jul 5-9, 1976

[5] D. Strottman, Phys. Rev. D 20, 748 (1979).

[6] H. J. Lipkin, Phys. Lett. B 195, 484 (1987).

[7] G. S. Adkins, C. R. Nappi and E. Witten, Nucl. Phys. B 228, 552 (1983).

[8] M. Chemtob, Nucl. Phys. B 256, 600 (1985).

[9] M. Praszalowicz, in Skymions and Anomalies, M. Jezabeck and M. Praszalowicz, eds., World Scientific (1987), p. 112 .

[10] D. Diakonov, V. Petrov and M. V. Polyakov, Z. Phys. A 359, 305 (1997) arXiv:hep-ph/9703373.

[11] See also H. Weigel, Eur. Phys. J. A 2, 391 (1998) arXiv:hep-ph/9804260.

[12] K. Hicks, private communication. 
[13] T. D. Cohen, arXiv:hep-ph/0309111 N. Itzhaki, I. R. Klebanov, P. Ouyang and L. Rastelli, arXiv:hep-ph/0309305

[14] F. E. Close and N. A. Tornqvist, J. Phys. G 28, R249 (2002) arXiv:hep-ph/0204205.

[15] R. L. Jaffe, Phys. Rev. D 15, 267, 281 (1977).

[16] M. Anselmino, E. Predazzi, S. Ekelin, S. Fredriksson and D. B. Lichtenberg, Rev. Mod. Phys. 65, 1199 (1993).

[17] K. Rajagopal and F. Wilczek, arXiv:hep-ph/0011333
[18] A. De Rujula, H. Georgi and S. L. Glashow, Phys. Rev. D 12, 147 (1975).

[19] M. G. Alford and R. L. Jaffe, Nucl. Phys. B 578, 367 (2000) arXiv:hep-lat/0001023.

[20] For a different analysis, which predicts instability of the $\Theta_{c}^{0}$ and $\Theta_{b}^{+}$, see M. Karliner and H. J. Lipkin, arXiv:hep-ph/0307343

[21] [NA49 Collaboration], arXiv:hep-ex/0310014 